\title{
Synthesis of Diads and Triads Derived from Carotenoids and Fullerene C60
}

\author{
E. N. Durantini ${ }^{1}$, Ana Moore ${ }^{2}$, Thomas A. Moore $^{2}$ and Devens Gust ${ }^{2}$ \\ ${ }^{1}$ Departamento de Química y Física, Universidad Nacional de Río Cuarto, Agencia Postal No 3, 5800 \\ Río Cuarto, Argentina \\ E-mail: edurantini@exa.unrc.edu.ar \\ ${ }^{2}$ Department of Chemistry and Biochemistry, Arizona State University, Tempe, AZ 85287-1604, USA
}

\begin{abstract}
A convenient procedure for the synthesis of supramolecules bearing carotenoids and fullerene C60 is reported. The amphipathic nature and the high yield of charge separation of these compounds make them candidates in the formation of transmembrane charge gradients.
\end{abstract}

\section{Introduction}

Photochemical and photophysical studies of fullerene C60 (buckminsterfullerene) have shown that C60 is a good electron-acceptor, has a low fluorescence and high triplet quantum yield (@100\%) [1]. In carotenoid-fullerene diads, the lowest excited singlet state of the fullerene is strongly quenched by electron transfer from the carotenoid moiety to generate the charge separated species $[2,3]$. The high yield of charge separation and the amphipathic nature of these supramolecules, make then a likely candidates for use in the formation of transmembrane charge gradients, producing proton transport across phospholipid membranes, upon absorption of light [4].

\section{Experimental}

All the products were characterized by ${ }^{1} \mathrm{HNMR}$ and MS spectroscopies. The precursor methanofullerenecarboxylic acid was prepared according to the method described in the literature [1]. The Wittig-Horner reactions were performed in $\mathrm{THF} / \mathrm{KOH}$ medium.

\section{Results and Discussion}

\section{Synthesis of Bifunctional Carotenoids}

Bifunctional carotenoids, substituted on both sides of the conjugate chain, were synthesized from crocetindial. An aminophenyl group was incorporated on one side of the chain by the Wittig reaction. 
In the other side, remains an aldehyde group, which was used for attach different structures by formation of a new double bond using Wittig-Horner reactions.

\section{C60 derivatives}

The methanofullerenecarboxylic acid (C60-acid) was synthesized from C60 using the method described by Diederich [1]. This compound is a versatile starting material for the preparation of amphipathic fullerene derivatives. The coupling reaction of carotenoids to $\mathrm{C} 60$-acid was performed using dicyclohexylcarbodiimide catalyzed by 1-hydroxybenzotriazole, 4-dimethylaminopyridine and triethylamine (44-50\% yields).

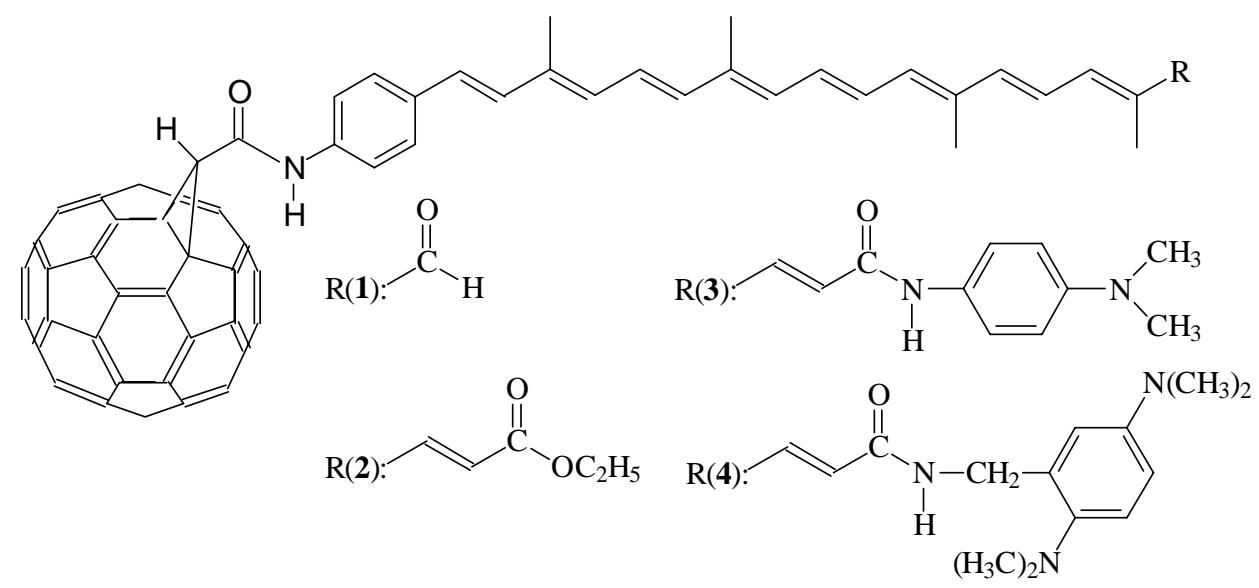

Acknowledgements: Authors are grateful to Fundación Antorchas, Consejo Nacional de Investigaciones Científicas y Técnicas of Argentina and National Science Foundation for financial support.

\section{References and Notes}

1. Diederich, F.; Whetten, R. L. Acc. Chem. Res. 1992, 25, 119.

2. Imahori, H.; Cardoso, S.; Tatman, D.; Lin, S.; Noss, L.; Seely, G.; Sereno, L.; Silber, J.; Moore, T. A.; Moore, A.L.; Gust D. Photochem. Photobiol. 1995, 62, 1009.

3. Tatman, D.; Durantini, E.N.; Moore, A.L.; Moore, T.A.; Gust, D. Photchem. Photobiol. 1997, 65.

4. Steinberg-Yfrach, G.; Rigaud, J-L.; Durantini, E. N.; Moore, A. L.; Gust, D.; Moore, T. A. Nature 1998, 392, 479. 